\title{
Strain engineering of the electroabsorption response in Ge/SiGe multiple quantum well heterostructures
}

\author{
L. Lever, ${ }^{1, *}$ Y. Hu, ${ }^{2}$ M. Myronov,${ }^{3}$ X. Liu,${ }^{3}$ N. Owens,${ }^{2}$ F. Y. Gardes,${ }^{2}$ I. P. Marko, ${ }^{2}$ \\ S. J. Sweeney, ${ }^{2}$ Z. Ikonić, ${ }^{1}$ D. R. Leadley, ${ }^{3}$ G. T. Reed, ${ }^{2}$ and R. W. Kelsall ${ }^{1}$ \\ ${ }^{1}$ Institute of Microwaves and Photonics, School of Electronic and Electrical Engineering, \\ University of Leeds, Leeds LS2 9JT, United Kingdom \\ ${ }^{2}$ Advanced Technology Institute, University of Surrey, Guildford GU2 7XH, United Kingdom \\ ${ }^{3}$ Department of Physics, University of Warwick, Coventry CV4 7AL, United Kingdom
}

Many fibre-optic telecommunications systems exploit the spectral 'window' at $1310 \mathrm{~nm}$, which corresponds to zero dispersion in standard single-mode fibres (SMFs). In particular, several passive optical network (PON) architectures use $1310 \mathrm{~nm}$ for upstream signals, ${ }^{1}$ and so compact, low-cost and low-power modulators operating at $1310 \mathrm{~nm}$ that can be integrated into Si electronicphotonic integrated circuits would be extremely desireable for futre fibre-to-the-home (FTTH) applications.

Ge/SiGe multiple quantum wells can be eptixially grown on $\mathrm{Si}$ wafers by exploiting a relaxed $\mathrm{SiGe}$ virtual substrate, and the quantum-confined Stark effect (QCSE) in this material system has been reported with a variety of well dimensions. ${ }^{2-4}$ Owing to the lattice mismatch between $\mathrm{Si}$ and $\mathrm{Ge}$, to achieve mechanical stability the alternating layers should be compressive/tensile strained, and the Ge fraction of the substrate chosen so that the multiple quantum well (MQW) stack is strain symmetrised to the virtual substrate.

As the Si fraction in the virtual substrate increases, so does the compressive strain in the Ge quantum wells. This compressive strain results in a blue shift of the direct absorption edge, and a suitable choice of well dimensions and substrate composition can be chosen to target a given operating wavelength for a MQW modulator. ${ }^{5}$

Existing Ge/SiGe MQW heterostructures have been reported using virtual substrates that are typically composed of $\mathrm{Si}_{0.1} \mathrm{Ge}_{0.9}$ (although heterostructures have also been reported grown on $\mathrm{Si}_{0.05} \mathrm{Ge}_{0.95}$ substrates). Here we



FIG. 1: Schematic diagram showing a cross section of the MQW $p-i-n$ photodiodes. The MQW layer consists of ten wells and eleven barrier, and is sandwiched between two intrinsic SiGe spacer layers in order to achieve a uniform electric field across each quantum well in the stack.



FIG. 2: Absorption spectra for the $14-\mathrm{nm} \mathrm{Ge} / \mathrm{Si}_{0.4} \mathrm{Ge}_{0.6}$ quantum wells at a range of applied biases. The light-hole exciton can be seen at approximately $1240 \mathrm{~nm}$ in the zero-field curve. This large light-hole/heavy-hole splitting is evidence of the large strain due to the high Si fraction of the virtual substrate.

demonstrate QCSE in Ge/SiGe heterostructures grown on $\mathrm{Si}_{0.2} \mathrm{Ge}_{0.8}$ virtual substrates, and report a significant blue shift of the direct absorption edge compared to existing structures.

The Ge/ $\mathrm{Si}_{0.4} \mathrm{Ge}_{0.6} \mathrm{MQW}$ heterostructures were grown using reduced pressure chemical vapour deposition (RPCVD) on a $\mathrm{Si}_{0.2} \mathrm{Ge}_{0.8}$ relaxed virtual substrate. Our virtual substrates were achieved using reverse linear grading from a relaxed Ge seed layer, which is grown on an Si substrate. ${ }^{6}$ Circular mesa devices were defined using optical lithography and reactive ion etching (RIE), and a $\mathrm{Ti} / \mathrm{Al}$ metal stack was deposited to form electrical contacts. A schematic diagram of the cross section of the devices is shown in Fig. 1. Absorption spectra were inferred from the wavelength-dependent photocurrent measured using a $100-\mathrm{W}$ xenon light source and a $3-\mathrm{nm}$-bandwidth monochromator.

Figure 2 shows absorption spectra for a 14-nm ten quantum well stack at a range of applied biases. The large strain results in a larger light-hole/heavy-hole splitting than is observed in similar systems grown on $\mathrm{Si}_{0.1} \mathrm{Ge}_{0.9}$ virtual substrates, as well as a blue shift of the zero field absorption edge by approximately $70 \mathrm{~nm}$ 




FIG. 3: Absorption spectra for the $9-\mathrm{nm} \mathrm{Ge} / \mathrm{Si}_{0.4} \mathrm{Ge}_{0.6}$ quantum wells.

compared with the data for 14-nm quantum wells on a $\mathrm{Si}_{0.1} \mathrm{Ge}_{0.9}$ virtual substrate reported in Ref. 7 . The exciton peaks are well resolved and a Stark shift is clearly observed.

Figure 3 shows absorption spectra for a 9-nm ten quantum well stack at a range of applied biases. The increased confinement energy results in a further blue shift of the absorption edge to approximately $1240 \mathrm{~nm}$. The excitonic peaks are less well defined than in the spectra from the 14-nm sample. This may be due to increased lifetime broadening of the excitons. The reduction of the dimensions of the quantum wells increases in confinement energy of the $\Gamma$-valley electrons more than those of the $L$-valley electron states because of the smaller $\Gamma$-valley effective mass. This means that there are more $L$-valley states available to scatter into, reducing the lifetime of the $\Gamma$-valley electron state. However, because the confinement energy has increased, we can also expect a larger inhomogeneous braodening component due to fluctuations in the dimensions of the quantum well throughout the mesa device.

The observed Stark shift is much smaller for the 9-nm quantum well heterostructure than for the 14-nm quantum wells, which can be explained by a combination of the increased exciton linewidth and the fact that the dimensions of the quantum well are reduced compared with the spectra in Fig. 2. Note that a significant increase in the indirect absorption is not observed when we use structures where the absorption edge is at shorter wavelengths. The relative magnitude of the sub-bandgap absorption does not significantly alter when we move to shorter wavelengths; however, the excitonic lineshape does.

We have demonstrated that strain engineering can be used to target wavelengths close to the commercially important $1310 \mathrm{~nm}$ spectral 'window' for electroabsorption modulation using Ge/SiGe MQW heterostructures.

* Electronic address: 1.j.m.lever@leeds.ac.uk

1 C. Lam, Passive Optical Networks Principles and Practice. Elsevier, 2007.

${ }^{2}$ Y.-H. Kuo, Y. K. Lee, Y. Ge, S. Ren, J. E. Roth, T. I. Kamins, D. A. B. Miller, and J. S. Harris, "Strong quantumconfined Stark effect in germanium quantum-well structures on silicon," Nature, vol. 437, pp. 1334-1336, 2005.

3 P. Chaisakul, D. Marris-Morini, G. Isella, D. Chrastina, X. L. Roux, E. Gatti, S. Edmond, J. Osmond, E. Cassan, and L. Vivien, "Quantum-confined stark effect measurements in Ge/SiGe quantum-well structures," Optics Letters, vol. 35, no. 17, pp. 2913-2915, Sep 2010.

${ }^{4}$ R. K. Schaevitz, J. E. Roth, S. Ren, O. Fidaner, and D. A. Miller, "Material properties of Si-Ge/Ge quantum wells," IEEE Journal of selected topics in quantum electronics, vol. 14, pp. 1082-1089, 2008.

${ }^{5}$ L. Lever, Z. Ikonić and, A. Valavanis, J. Cooper, and R. Kelsall, "Design of Ge-SiGe quantum-confined stark effect electroabsorption heterostructures for CMOS compatible photonics," Lightwave Technology, Journal of, vol. 28, no. 22, pp. $3273-3281,2010$.

6 V. A. Shah, A. Dobbie, M. Myronov, D. J. F. Fulgoni, L. J. Nash, and D. R. Leadley, "Reverse graded relaxed buffers for high Ge content SiGe virtual substrates," $A p$ plied Physics Letters, vol. 93, no. 19, p. 192103, 2008.

7 C. Lange, N. S. Köster, S. Chatterjee, H. Sigg, D. Chrastina, G. Isella, H. von Känel, M. Schäfer, M. Kira, and S. W. Koch, "Ultrafast nonlinear optical response of photoexcited $\mathrm{Ge} / \mathrm{SiGe}$ quantum wells: Evidence for a femtosecond transient population inversion," Physical Review B, vol. 79, no. 20, p. 201306, May 2009. 